\title{
A murine colitis model developed using a combination of dextran sulfate sodium and Citrobacter rodentium
}

\author{
Jin-Il Park ${ }^{1,2}$, Sun-Min Seo ${ }^{1}$, Jong-Hyung Park ${ }^{1}$, \\ Hee-Yeon Jeon ${ }^{1,3}$, Jun-Young Kim ${ }^{1}$, \\ Seung-Hyun Ryu ${ }^{1,4}$, and Yang-Kyu Choi ${ }^{1 *}$ \\ ${ }^{1}$ Department of Laboratory Animal Medicine, College of Veterinary \\ Medicine, Konkuk University, Seoul 05029, Republic of Korea \\ ${ }^{2}$ ViroMed Co. Ltd., Seoul 08826, Republic of Korea \\ ${ }^{3}$ Department of Core Research Laboratory, Clinical Research Institute, \\ Kyung Hee University Hospital at Gangdong, Seoul 05278, \\ Republic of Korea \\ ${ }^{4}$ Samjin Pharmaceutical Co., Ltd., Seoul 04054, Republic of Korea \\ (Received Nov 16, 2017 / Revised Jan 29, 2018 / Accepted Feb 4, 2018)
}

Adult mice were treated with dextran sulfate sodium (DSS) and infected with Citrobacter rodentium for developing a novel murine colitis model. C57BL/6N mice (7-week-old) were divided into four groups. Each group composed of control, dextran sodium sulfate-treated (DSS), C. rodentiuminfected $(\mathrm{CT})$, and DSS-treated and C. rodentium-infected (DSS-CT) mice. The DSS group was administered 1\% DSS in drinking water for 7 days. The CT group was supplied with normal drinking water for 7 days and subsequently infected with $C$. rodentium via oral gavage. The DSS-CT group was supplied with $1 \%$ DSS in drinking water for 7 days and subsequently infected with $C$. rodentium via oral gavage. The mice were sacrificed 10 days after the induction of $C$. rodentium infection. The DSS-CT group displayed significantly shorter colon length, higher spleen to body weight ratio, and higher histopathological score compared to the other three groups. The mRNA expression levels of tumor necrosis factor (TNF)- $\alpha$ and interferon (INF)- $\gamma$ were significantly upregulated; however, those of interleukin (IL)- 6 and IL-10 were significantly downregulated in the DSS-CT group than in the control group. These results demonstrated that a combination of low DSS concentration (1\%) and C. rodentium infection could effectively induce inflammatory bowel disease (IBD) in mice. This may potentially be used as a novel IBD model, in which colitis is induced in mice by the combination of a chemical and a pathogen.

Keywords: Citrobacter rodentium, colitis, dextran sulfate sodium

\section{Introduction}

Inflammatory bowel disease (IBD), such as ulcerative colitis (UC) and Crohn's disease (CD), is the most important type of human enteritis that is characterized by chronic, relapsing, and recurrent inflammatory conditions, resulting from chronic dysregulation of the mucosal immune system in the gastrointestinal tract (Bauer et al., 2010). The exact mechanism underlying the pathogenesis of IBD is unknown; however, it is widely known that infection, immunological abnormalities, genetics, and environmental factors are important determinants of IBD (Kwon et al., 2005).

In the past few decades, numerous animal models of IBD have been developed including spontaneous colitis models, adoptive transfer models, genetically modified models, and inducible colitis models (Perše and Cerar, 2012). Due to their simplicity in induction and reproducibility, the chemically induced murine intestinal inflammation models developed using dextran sulfate sodium (DSS), trinitrobenzene sulfonic acid, oxazolone, acetic acid, and nonsteroidal anti-inflammatory drugs are widely used (Randhawa et al., 2014). Especially, the DSS-induced IBD model is widely used because of its simplicity and similarities with human IBD (Kim et al., 2012; Chassaing et al., 2014). DSS is a water-soluble, negatively charged, sulfated polysaccharide, with molecular weight ranging from 5 to $1,400 \mathrm{kDa}$ (Chassaing et al., 2014). The molecular weight of DSS is an important factor in the induction of colitis in mice and rats, and most severe colitis results from the use of $\sim 40-50 \mathrm{kDa}$ DSS (Hirono et al., 1983; Axelsson et al., 1996; Kitajima et al., 2000). Generally, DSS at a concentration of $2-5 \%$ is administrated after dissolving it in drinking water, which produces either acute or chronic colitis depending on the experimental protocol (Chassaing et al., 2014). Administration of DSS results in symptoms like loose stool, diarrhea, bloody feces, body weight loss, mucosal ulceration, and shortening of the colon and rectum (Kwon et al., 2005). In DSS-induced colitis, cytokine levels of tumor necrosis factor (TNF)- $\alpha$, interleukin (IL)-1 $\beta$, IL-6, IL-10, and IL-17 were upregulated (Randhawa et al., 2014).

Citrobacter rodentium, previously known as Citrobacter freundii biotype 4280 or Citrobacter species 9, is a Gramnegative rod-shaped bacterium and the causative agent of transmissible murine colonic hyperplasia. It is a naturally occurring pathogen in the laboratory mice. These mice are characterized by epithelial cell hyperproliferation in their descending colons (Luperchio and Schauer, 2001). C. rodentium is transmitted by the fecal-oral route and colonizes the gastrointestinal tract, following migration to the cecum and colon, via the formation of attaching and effacing (A/E) lesions (Alipour et al., 2013). Enteropathogenic Escherichia
${ }^{\star}$ For correspondence. E-mail: yangkyuc@konkuk.ac.kr; Tel.: +82-2-20496113; Fax: +82-2-450-3037

Copyright (c) 2018, The Microbiological Society of Korea 
(A)

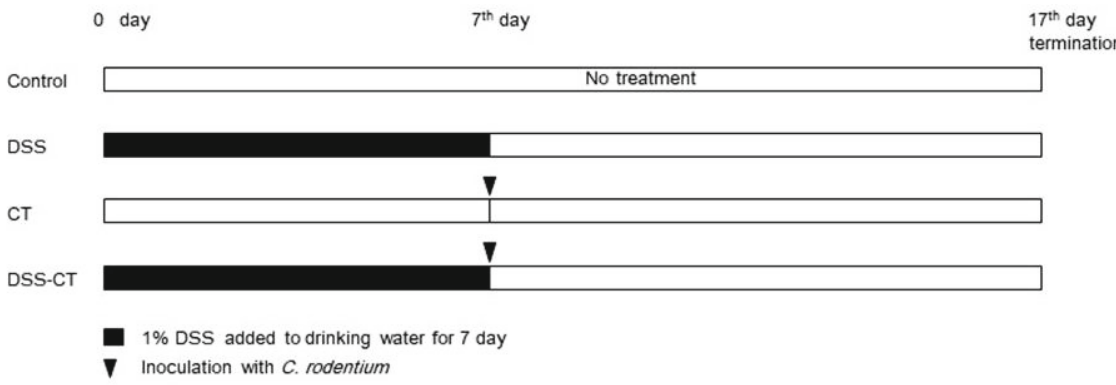

(B)

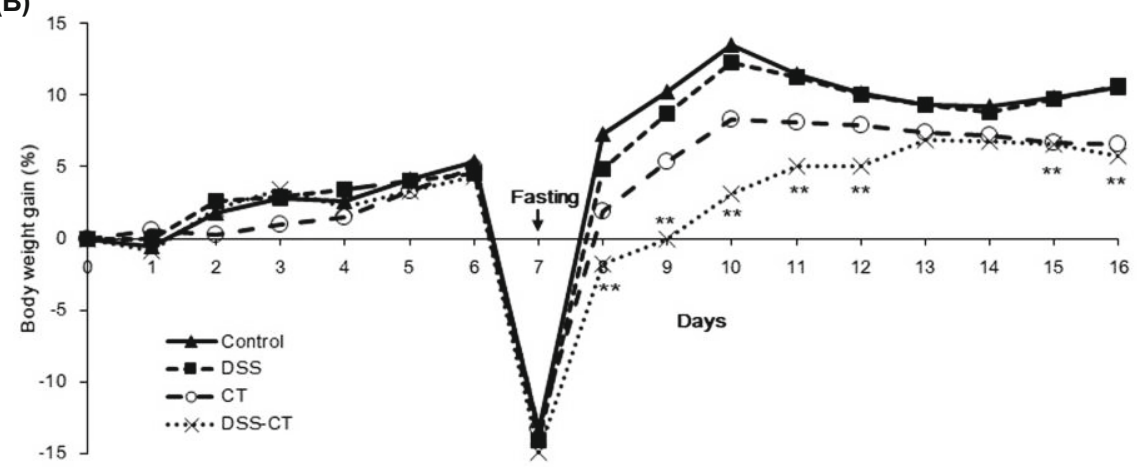

Fig. 1. Experimental procedure for inducing colitis and the measurement of body weight changes in mice. (A) Forty mice were divided into four groups (10 mice/group) and pretreated with dextran sulfate sodium (DSS), or normal drinking water for 1 week, and subsequently inoculated with $C$. rodentium or fresh LB broth. The mice were sacrificed 10 days after the induction of $C$. rodentium infection. (B) The body weight was measured every day and was plotted as the percentage of the initial body weight of the mouse. The data are expressed as the mean $\left({ }^{*} P<0.01\right.$, compared to the control and DSS groups). coli (EPEC; the etiological agent of infantile diarrhea) and enterohemorrhagic E. coli (EHEC; the causative agent of hemorrhagic diarrhea and hemolytic uretic syndrome) are members of the A/E family (Deng et al., 2001).

Whereas both EPEC and EHEC are poorly pathogenic in the mouse intestinal epithelial cells, $C$. rodentium is a much better A/E-lesion-forming pathogen compared to E. coli. C. rodentium infection results in the depletion of goblet cells, crypt cell hyperplasia, inflammatory cell infiltration, and significant intestinal barrier disruption in mouse models (Luperchio and Schauer, 2001; Mundy et al., 2005). C. rodentium-infected mice develop various pathologies depending on their age, genetic background, diet, and indigenous microbiota (Mundy et al., 2005). C. rodentium infection in the suckling and immunodeficient mice results in the development of colitis, characterized by diarrhea, rectal prolapse, and other clinical signs including retarded growth, listlessness, dehydration, and mortality (in severe cases) (Luperchio and Schauer, 2001; Ryu et al., 2016). In contrast, the infection in adult mice is subclinical and self-limiting, resulting in considerably less morbidity or mortality (Maaser et al., 2004). Therefore, it is difficult to induce colitis using C. rodentium in adult mice.

Several studies related to the development of colitis models in mice using chemicals or pathogens have been reported; however, no studies have reported their simultaneous usage. The main purpose of this study was not to induce colitis by using chemicals or pathogens alone, but by using them together. In this study, a novel colitis mouse model was developed by administration of low concentration (1\%) of DSS for 7 days, subsequently followed by infection with C. rodentium.

\section{Materials and Methods}

\section{Animals}

All procedures were approved by the Institutional Animal Care and Use Committee of Konkuk University. Six-weekold female C57BL/6N mice were purchased from Koatech Co., Ltd. The mice were acclimatized for one week in the laboratory animal breeding room of the College of Veterinary Medicine, Konkuk University. All mice were housed in sterile polycarbonate cages with wire lids, sterile bedding, and filter cage tops, under controlled pathogen-free conditions with a $12: 12$-h light:dark cycle at $22 \pm 2{ }^{\circ} \mathrm{C}$ and $50 \pm$ $10 \%$ humidity. The mice were fed sterilized food and water ad libitum.

\section{Bacterial strain and DSS administration}

C. rodentium strain DBS 100 (ATCC 51459) was inoculated on Luria-Bertani (LB) agar and allowed to grow overnight at $37^{\circ} \mathrm{C}$. Subsequently, it was cultured in LB broth overnight at $37^{\circ} \mathrm{C}$. The culture broth was centrifuged at $1,200 \times g$ for $10 \mathrm{~min}$. After centrifugation, the bacterial pellet was resuspended in fresh LB medium. The mice were infected with $0.2 \mathrm{ml}$ of LB medium containing $1.6 \times 10^{8} \mathrm{CFU}$ via oral gavage. The $\mathrm{CFU} / \mathrm{ml}$ were determined by plating serial dilutions. In this study, the molecular weight range of DSS (MP Biomedicals Inc.) used was $36-50 \mathrm{kDa}$. Low concentration of DSS solution (1\%) was prepared in sterile drinking water and administrated ad libitum.

\section{Experimental design}

After one week of acclimation, the 7-week-old mice were divided into four groups. The mice were administered $1 \%$ DSS or normal drinking water for 1 week and infected with 
Table 1. Histopathologic score to quantify the degree of intestinal inflammation

\begin{tabular}{|c|c|c|c|c|c|}
\hline \multirow{2}{*}{ Criterion } & \multicolumn{5}{|c|}{ Score } \\
\hline & 0 & 1 & 2 & 3 & 4 \\
\hline Loss of goblet cells & None & $\uparrow$ & $\uparrow \uparrow$ & $\uparrow \uparrow \uparrow$ & $\uparrow \uparrow \uparrow \uparrow$ \\
\hline Mucosa thickening & None & $\uparrow$ & $\uparrow \uparrow$ & $\uparrow \uparrow \uparrow$ & $\uparrow \uparrow \uparrow \uparrow$ \\
\hline Inflammatory cells in mucosa & None & $\uparrow$ & $\uparrow \uparrow$ & $\uparrow \uparrow \uparrow$ & $\uparrow \uparrow \uparrow \uparrow$ \\
\hline Inflammatory cells in submucosa & None & $\uparrow$ & $\uparrow \uparrow$ & $\uparrow \uparrow \uparrow$ & $\uparrow \uparrow \uparrow \uparrow$ \\
\hline
\end{tabular}

C. rodentium via oral gavage. They were fasted for $12 \mathrm{~h}$ prior to $C$. rodentium infection. The control group was supplied with normal drinking water for 7 days and inoculated with fresh LB broth on the seventh day after the start of the experiment. The DSS group was administered 1\% DSS in normal drinking water for 7 days and inoculated with fresh LB broth on the seventh day as above. The CT group was administered normal drinking water for 7 days and infected with $C$. rodentium on the seventh day via oral gavage. The DSS-CT group was administered 1\% DSS in normal drinking water for 7 days and infected with $C$. rodentium on the seventh day via oral gavage (Fig. 1A). The body weights of mice were recorded every day. The mice were euthanized seventeen days after the experiment. The spleen was removed, weighed, and stored at $-70^{\circ} \mathrm{C}$ until further analysis. The entire large intestine was collected and the length of colon was measured.

\section{Histopathological evaluation of colitis}

Portions of mid and distal colon were fixed in $10 \%$ buffered formalin, dehydrated using a gradient of ethanol, clarified with xylene, and embedded in paraffin. The embedded paraffin blocks were cut into $4-\mu \mathrm{m}$ sections and stained with hematoxylin and eosin (H\&E). Quantification for histopathology was performed from the images using the MetaMorph 7.5 computerized image analysis software (Molecular Devices). The selected sections were scored by two blinded investigators. Colonic histopathology was scored as described previously (Rath et al., 1996), with slight modifications (Table 1). The tissue sections were assessed for goblet cell depletion (scored based on the number of goblet cells per high-power field at $400 \times$ magnification, where $0,>240 ; 1,181-240 ; 2$, 121-180; 3, 61-120; and 4, <60 cells), mucosal thickening $(0,<200 ; 1,201-300 ; 2,301-400 ; 3,400-500$; and 4, > 500 $\mu \mathrm{m}$ ), inflammatory cells in mucosa or submucosa (scored based on the number of inflammatory cells in mucosa or submucosa per high-power field at $400 \times$ magnification, where
$0,<50 ; 1,50-100 ; 2,51-150 ; 3,151-200$; and 4, $>200$ cells).

\section{Extraction of RNA and cDNA synthesis}

Total RNA was extracted from the stored spleen tissue using the RNeasy Mini kit (Qiagen), according to the manufacturer's instructions. RNA was quantitated by determining the absorbance or optical density (OD) at $260 \mathrm{~nm}$. Further, it was assessed for purity by measuring the ratio of absorbance at 260 and $280 \mathrm{~nm}\left(\mathrm{~A}_{260 / 280}\right)$ and direct examination of RNA bands on $1.5 \%$ agarose gels. Equal amounts of RNA were reverse transcribed into cDNA using the M-MLV reverse transcriptase (Invitrogen). Briefly, $5 \mu \mathrm{g}$ of total RNA extracted from a tissue was reverse transcribed in $20 \mu \mathrm{l}$ of reaction mixture containing the RT buffer, oligo(dT) $)_{12-18}$ primer $(500 \mu \mathrm{g} / \mathrm{ml}, 1 \mu \mathrm{l})$, dNTP mix $(10 \mathrm{mM}, 1 \mu \mathrm{l})$, RNaseOUT ${ }^{\mathrm{TM}}$ $(1 \mu \mathrm{l}), 5 \times$ first-strand buffer $(4 \mu \mathrm{l})$, DTT $(0.1 \mathrm{M}, 2 \mu \mathrm{l})$, and MMLV reverse transcriptase $(1 \mu \mathrm{l})$. The cDNA was used as a template for real-time PCR.

\section{Real-time PCR}

Real-time PCR was performed using the Bio-Rad CFX96 real-time PCR detection system (Bio-Rad), according to the manufacturer's instructions. The TaqMan ${ }^{\mathrm{TM}}$ fluorogenic probes and PCR primers for TNF- $\alpha$, INF- $\gamma$, IL-6, IL-10, IL-1 $\beta$, monocyte chemoattractant protein-1 (MCP-1), and GAPDH were obtained from Bio-Rad. The threshold cycle $\left(C_{t}\right)$, which correlates inversely with the target mRNA expression level, was measured as the cycle number at which the reporter fluorescence emission increased above the threshold level. The relative changes in TNF- $\alpha$, IFN- $\gamma$, IL- 6 , IL-10, IL-1 $\beta$, and MCP-1 mRNA levels were normalized with the levels of GAPDH mRNA in the same samples.

\section{Statistical analysis}

All data obtained in this study were analyzed using the GraphPad Prism 5.0 software (GraphPad Software, Inc.). Data were
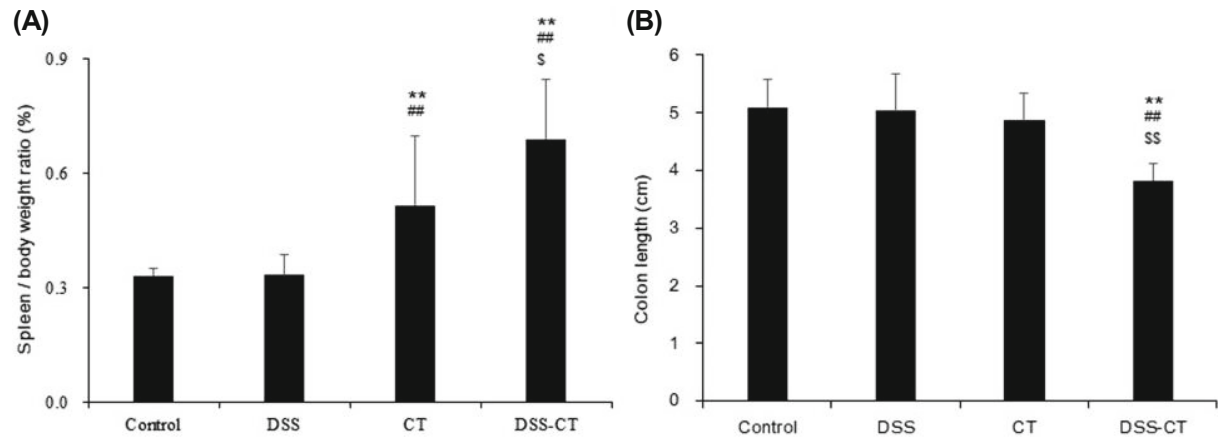

Fig. 2. Weight of the spleen and length of the colon. Body weight, spleen weight, and colon length were measured after the experiment ( $\mathrm{n}=10$ mice per group). (A) Spleen to body weight ratio. (B) Colon length. The data are expressed as the mean $\pm \mathrm{SD}\left({ }^{* *} P<0.01\right.$, compared to control group; ${ }^{\# \#} P<0.01$, compared to DSS group; ${ }^{\$} P<0.05$ and ${ }^{\$ \$} P<0.01$, compared to the CT group). 
expressed as the mean \pm standard deviation (SD). The results were considered to be statistically significant at $P<0.05$ and $P<0.01$.

\section{Results}

Combination of DSS administration and C. rodentium infection decreases the gain in body weight

Body weights of the mice were measured every day. For each mouse, the change in body weight was calculated as the percentage of its initial weight (Fig. 1B). In all the groups, the gain in body weight gradually increased by about $4 \%$, until 6 days after the start of the experiment. Subsequently, on the seventh day, it decreased about $14 \%$ in all the groups due to fasting. The mice were fasted for $12 \mathrm{~h}$ to increase the infectivity of $C$. rodentium. No differences in the gain of body weight were observed among all the groups prior to $C$. rodentium infection. However, after $C$. rodentium infection, the body weight gain in the DSS-CT group was significantly $(P$ $<0.01)$ lower than that in the control and DSS groups.

\section{Combination of DSS and C. rodentium treatment increases} the spleen to body weight ratio and decreases colon length

The DSS-CT group displayed significantly $(P<0.05)$ higher spleen to body weight ratio $(0.69 \pm 0.16 \%)$ compared to the CT group $(0.52 \pm 0.18 \%)$, and significantly $(P<0.01)$ higher spleen to body weight ratio than the control $(0.33 \pm 0.02 \%)$ and DSS $(0.33 \pm 0.05 \%)$ groups (Fig. $2 \mathrm{~A})$. The ratio of spleen to body weight was also significantly $(P<0.01)$ higher in the $\mathrm{CT}$ group than in the control and DSS groups. The entire colon length was also measured for all the mice after sacrificing them. The DSS-CT group displayed significantly $(P$ $<0.01)$ shorter colon length $(3.83 \pm 0.3 \mathrm{~cm})$ compared to the control $(5.1 \pm 0.48 \mathrm{~cm})$, DSS $(5.03 \pm 0.65 \mathrm{~cm})$, and CT $(4.87 \pm 0.47 \mathrm{~cm})$ groups (Fig. $2 \mathrm{~B})$. No difference in the co-
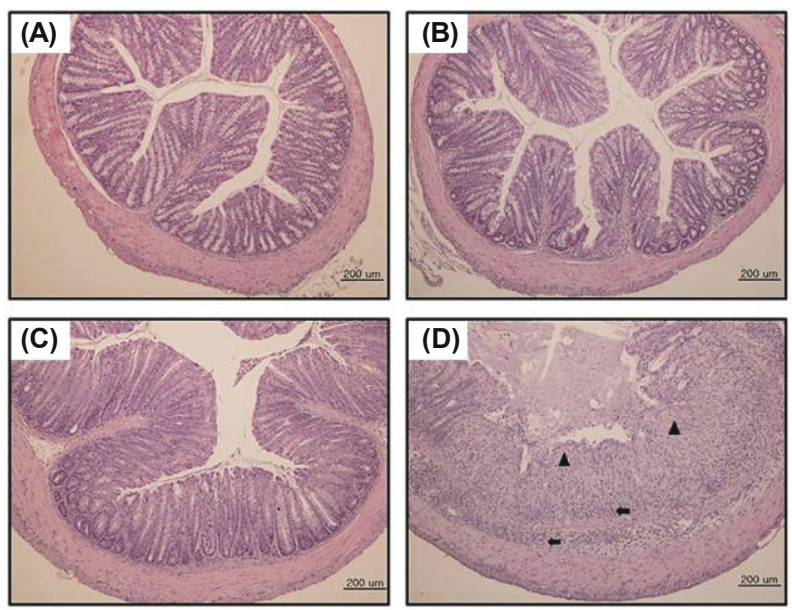

Fig. 3. Representative images of histopathological lesions in the colon of control (A), DSS (B), CT (C), and DSS-CT (D) group mice. Depletion of goblet cells (arrowheads) and inflammatory cell infiltration (arrows) were observed in the DSS-CT group (H\&E stain). Scale bar $=200 \mu \mathrm{m}$.

lon length was observed among the control, DSS, and CT groups.

\section{Combination of DSS and C. rodentium treatment decreases histopathological lesions in the colon}

Disruption and hyperplasia of mucosa are important indicators of colitis. In the DSS-CT group, histopathological lesions in the colon were characterized by loss of goblet cells, mucosal thickening, and infiltration of inflammatory cells in the mucosa and submucosa (Fig. 3D and Supplementary data Fig. S1). The histopathological lesions were examined independently by two pathologists and evaluated using the scoring system. In the DSS and CT groups, loss of goblet cells, mucosal thickening, and infiltration of inflammatory
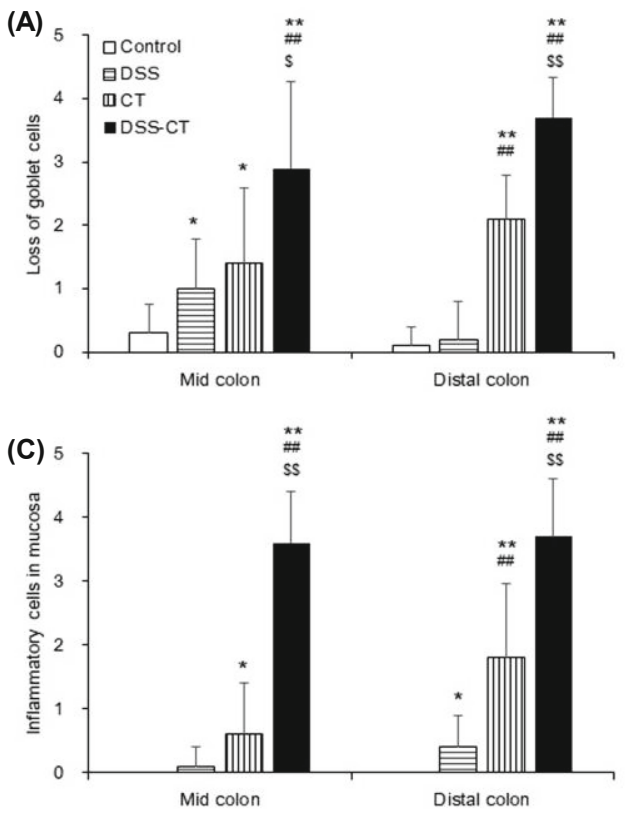
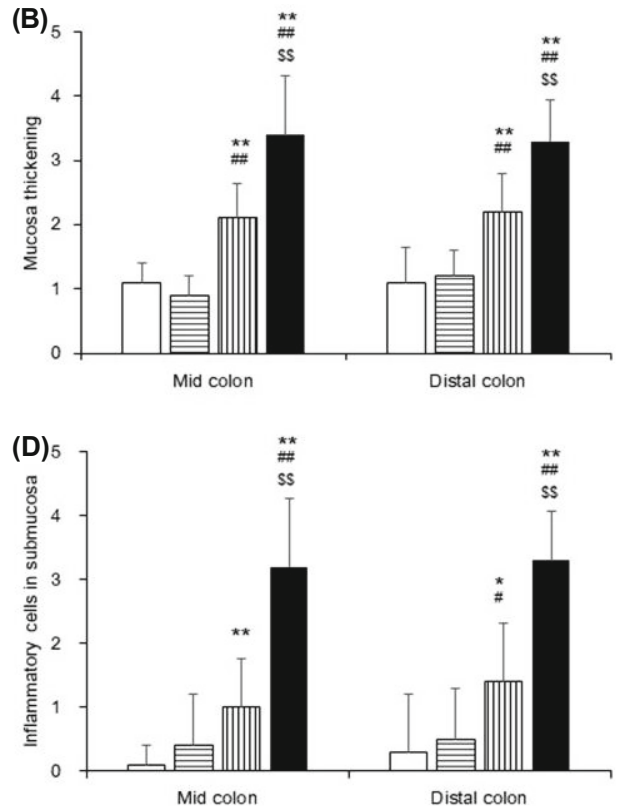

Fig. 4. Total histopathological scoring for goblet cell loss (A), mucosal thickening (B), inflammatory cells in mucosa (C), and submucosa (D) in the mid and distal colon regions of mice. The scores are expressed as the mean $\pm \mathrm{SD}\left({ }^{*} P<\right.$ 0.05 and ${ }^{* *} P<0.01$, compared to the control group; ${ }^{*} P<0.05$ and ${ }^{\# \#} P<0.01$, compared to the DSS group; ${ }^{\$} P<0.05$ and ${ }^{\$ \$} P<0.01$, compared to the CT group). 


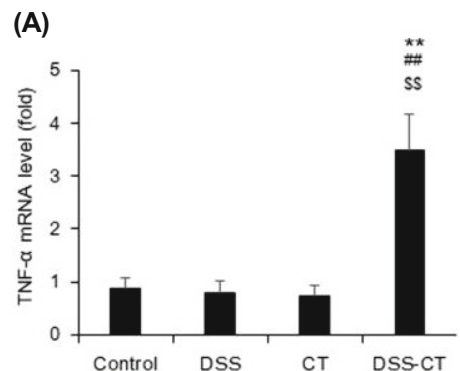

(D)

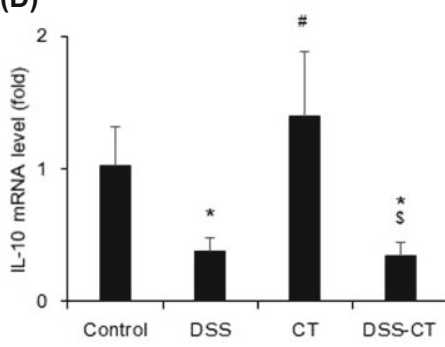

(B)

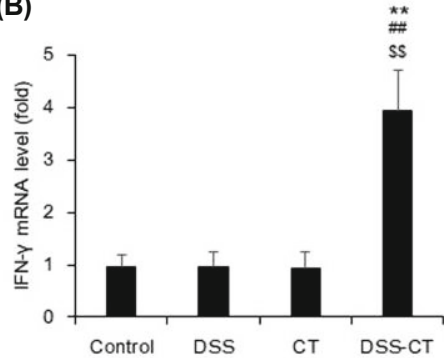

(E)

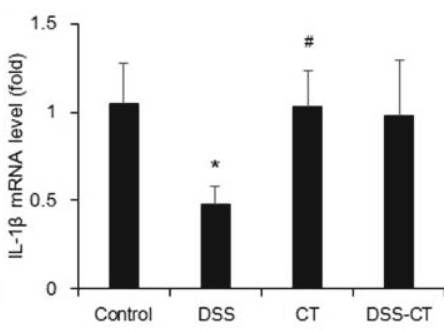

(C)

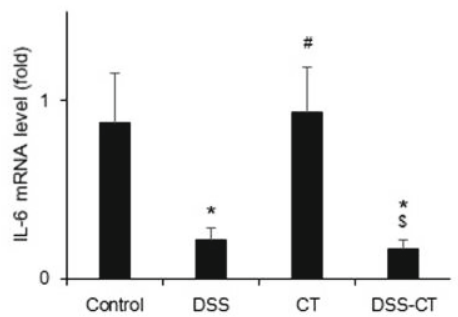

(F)

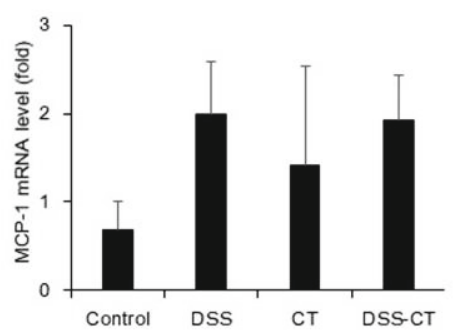

Fig. 5. mRNA expression of cytokine and chemokine genes in the spleen of control, DSS, CT, and DSS-CT group mice. mRNA expression levels of TNF- $\alpha$ (A), IFN- $\gamma$ (B), IL-6 (C), IL-10 (D), IL-1 $\beta$ (E), and MCP-1 were measured using real-time PCR. The mRNA expression levels were normalized with the GAPDH mRNA levels in the same samples. The scores were expressed as the mean \pm standard error $\left(^{*} P<0.05\right.$ and ${ }^{* *} P<0.01$, compared to the control group; ${ }^{\#} P<0.05$ and ${ }^{\# \#} P<0.01$, compared to the DSS group; ${ }^{\$} P<0.05$ and ${ }^{\$ \$} P<0.01$, compared to the CT group).

cells slightly increased compared to that in the control group. All four histopathological scores, including loss of goblet cells, mucosal thickening, and infiltration of inflammatory cells in the mucosa and submucosa, were significantly $(P<$ 0.05 ) higher in the mid and distal regions of the colon in the DSS-CT group compared to those in the other three groups (Fig. 4). The CT group also displayed significantly $(P<0.05)$ higher histopathological scores in the distal colon region compared to the control and DSS groups (Fig. 4). In contrast to the distal colon region of the CT group, the mid colon region displayed significantly $(P<0.05)$ higher histopathological scores than the control group. The total histopathological score was expressed as the sum of each individual histopathological score. The total histopathological scores for the mid and distal colon regions in the DSS-CT group (13.1 \pm 2.7 and $14 \pm 1.1$, respectively) were significantly $(P<0.01)$ higher compared to those for the other three groups. The total histopathological scores for the mid and distal colon regions in the CT group $(5.1 \pm 2.1$ and $7.5 \pm 2.2$, respectively) were also significantly $(P<0.01)$ higher than those for the control and DSS groups. No difference in the total histopathological score for the mid and distal colon regions was observed between the control ( $1.5 \pm 0.7$ and $1.5 \pm 1.3$, respectively) and DSS ( $2.4 \pm 1.4$ and $2.3 \pm 1.5$, respectively) groups.

\section{Combination of DSS and C. rodentium treatment increases the mRNA expression levels of inflammatory cytokines}

The mRNA expression levels of TNF- $\alpha$, IFN- $\gamma$, IL-6, IL-10, IL- $1 \beta$, and MCP-1 were evaluated in the spleen using realtime PCR. The mRNA expression levels of the proinflammatory cytokines, including TNF- $\alpha$ and IFN- $\gamma$, were significantly $(P<0.01)$ higher in the DSS-CT group compared to the other three groups (Fig. 5A and B). On the other hand, the mRNA expression levels of the anti-inflammatory cyto- kines, such as IL-6 and IL-10, were significantly $(P<0.05)$ lower in the DSS-CT group compared to those in the control and CT groups (Fig. 5C and D). Interestingly, compared to the control group, the mRNA expression levels of IL-6 and IL-10 did not change in the CT group; however, the levels significantly $(P<0.05)$ decreased in the DSS group (Fig. 5C and D). The mRNA expression level of IL- $1 \beta$ was significantly $(P<0.05)$ lower in the DSS group than in the control group. The mRNA expression of MCF-1 was higher in the other groups than in the control group; however, no significant differences were observed between the groups themselves (Fig. 5E and F).

\section{Discussion}

Although numerous colitis models induced using DSS have been developed, susceptibility to DSS depends on the species of animal used. In mice and rats, colitis develops primarily in the colon; however, in guinea pigs, the lesions develop mainly in the cecum (Okayasu et al., 1990; Iwanaga et al., 1994; Hoshi et al., 1996). Additionally, the symptoms of colitis also vary according to the molecular weight of DSS used (Chassaing et al., 2014). Acute or chronic colitis is usually induced by continuous administration of 2-5\% DSS for 4-9 days or by cyclic DSS treatment (Kwon et al., 2005; Perše and Cerar, 2012). We used both, a chemical and a pathogen, to induce murine colitis. Unlike previous studies, we treated mice with a very low dose $(1 \%)$ of DSS and C. rodentium. Thus, in this study, we established a novel model for IBD induced using both DSS and C. rodentium.

IBD is becoming increasingly prevalent and affects about 90-300 per 100,000 people worldwide (Casati and Toner, 2000). Although it occurs at any age, the peak age for the on- 
set of IBD is 15 to 30 years. The symptoms of IBD include chronic uncontrolled inflammation of the intestinal mucosa, which can affect all parts of the gastrointestinal tract (Andres and Friedman, 1999; Hanauer, 2006). However, the mechanism by which DSS induces colitis has not yet been elucidated. Interestingly, various studies have shown that DSS administration induces toxic effects on the colonic epithelium, causes alteration of the luminal bacterial flora, and activation of T cell-, B cell-, and macrophage-mediated inflammatory responses (Okayasu et al., 1990; Yamada et al., 1992; Ohkusa et al., 1995).

The clinically significant pathogenic strains EPEC and EHEC, have also been linked to IBD. EPEC and EHEC are known to induce $\mathrm{A} / \mathrm{E}$ lesions, which intimately attach to the apical host cell membrane of the cecal and colonic epithelium, thereby forming a non-invasive pedestal-like structure (Eckmann, 2006). However, mice are not susceptible to EPEC and EPEC infections. C. rodentium, an A/E pathogen, is the causative agent for transmissible murine colonic hyperplasia, a naturally occurring disease in mice (Luperchio and Schauer, 2001; Mundy et al., 2005). Oral C. rodentium infection in mice causes transient colonization of the cecum, progresses to the distal colon 2 to 3 days after infection, and peaks after 1 week. Subsequently, the infection gets cleared over the next 2-3 weeks (MacDonald et al., 2003; Eckmann, 2006). C. rodentium-induced typhlitis or colitis is difficult to induce in adult mice and some mouse strains, which are resistant to C. rodentium infection (Simmons et al., 2002).

To establish a novel IBD model in adult mice, we determined the percent of DSS and optimum dose of $C$. rodentium by conducting preliminary experiments. Experimental colitis in adult mice was not induced by administration of $0.5 \%$ DSS for 1 week, followed by infection with a high-dose $\left(1.6 \times 10^{9} \mathrm{CFU}\right)$ of $C$. rodentium. Following pretreatment with $1 \%$ DSS and infection using $1.6 \times 10^{9} \mathrm{CFU}$ of $C$. rodentium, a successful IBD model was not established because $60 \%$ mice died (Supplementary data Fig. S2). In this study, we successfully developed a colitis model using 7-week-old C57BL/6N adult mice. These mice were pretreated with $1 \%$ DSS for 7 days, and subsequently infected with $1.6 \times 10^{8}$ CFU of $C$. rodentium.

After the induction of $C$. rodentium infection, the gain in body weight significantly reduced in the DSS-CT group compared that in the control group. However, no significant difference in the gain in body weight was observed between the DSS or CT group compared to the control group. Unlike previous studies (Wu et al., 2008; Knod et al., 2014), in this study, body weight increased steadily, except during the fasting period, even in the DSS-CT group because we used only $1 \%$ DSS and a low dose of $C$. rodentium. Hypertrophy of spleen, hyperplasia, and shortening of the large intestine have been observed in the TLR2-deficient mice infected with C. rodentium (Gibson et al., 2008). Consistent with the previous reports, in the DSS-CT group, the spleen to body weight ratio significantly increased and colon length significantly shortened compared to the other three groups. Disrupted cell morphological characteristics were observed in the C. rodentium-infected mice, with loss of goblet cells, mucosal hyperplasia, and dramatic infiltration of inflammatory cells into the mucosa and submucosa (Luperchio and Schauer, 2001).
Histopathologically, loss of crypts, infiltration of inflammatory cells into the mucosa and submucosa, submucosal edema, erosion, and ulceration have been observed in murine colitis induced by DSS (Kitajima et al., 2000). Consistent with the previous observations, significant depletion of goblet cells, mucosal hyperplasia, and infiltration of inflammatory cells were observed in the mid and distal colon regions of the DSSCT group mice compared to the mice in the other three groups. These results suggested that $C$. rodentium infection, following administration of DSS, causes morphological alterations in the colon.

IFN- $\gamma$, predominantly produced by the $\mathrm{T}$ and natural killer (NK) cells, acts as mediator for macrophage activation and immunological responses (Flynn et al., 1993). Both TNF-a and IFN- $\gamma$ are proinflammatory cytokines that have diverse biological effects, including cytolysis, cytotoxicity, immunoregulation, cellular proliferation, and antiviral responses. Thus, their action further worsens the disease condition ( $\mathrm{Yu}$ et al., 2009). IL-10 is an anti-inflammatory cytokine predominantly produced by the monocytes, macrophages, and other cells including the type $2 \mathrm{~T}$ helper cells and $\mathrm{B}$ cells (Moore et al., 2001). Interestingly, IL-6, which is primarily produced by the T cells, B cells, monocytes, and PMNs, acts as a pro- and anti-inflammatory cytokine during immune responses (Scheller et al., 2011). The mRNA levels of TNF- $\alpha$, IFN- $\gamma$, IL- 6, IL-10, and IL- $1 \beta$ in the colon were significantly higher in the C57BL/6 mice with colitis that was induced using 2 or $2.5 \%$ DSS compared to the untreated control group mice (Ouyang et al., 2012; Sang et al., 2016). Contrary to the previous studies, the mRNA levels of TNF- $\alpha$ and IFN- $\gamma$ in the spleen did not change in the $1 \%$ DSS group; however, the mRNA levels of IL- 6 , IL-10, and IL- $1 \beta$ dramatically decreased in the $1 \%$ DSS group compared to the levels in the control group. This may be due to the concentration of DSS used and duration of its administration. In our colitis model induced using both DSS and $C$. rodentium, the mRNA expression levels of TNF- $\alpha$ and IFN- $\gamma$ were significantly higher in the DSS-CT group than in the other three groups. In contrast, the mRNA expression levels of IL- 6 and IL-10 were significantly lower in the DSS-CT group than in the control and CT groups. These data showed that low concentration of DSS, and not $C$. rodentium infection, inhibited the expression of cytokines, including IL-6 and IL-10. However, in our novel model, increase in the levels of proinflammatory cytokines, namely TNF- $\alpha$ and IFN- $\gamma$, was due to C. rodentium infection. These results suggested that in our colitis model, the immunological balance between pro- and antiinflammatory cytokines was maintained. Thus, this was a novel animal model of IBD.

In summary, we administrated a low concentration (1\%) of DSS to 7-week-old adult mice for 7 days, and subsequently infected them with $C$. rodentium via oral gavage. The mice showed less body weight gain, shortened colon length, increased spleen weight, and severe histopathological lesions (including depletion of goblet cells, mucosal hyperplasia, and inflammatory cell infiltration). The expression levels of TNF- $\alpha$ and IFN- $\gamma$ increased, and those of IL- 6 and IL-10 decreased in the DSS-CT group compared to the control group. In conclusion, a novel murine IBD model was effectively developed by the combination of low concentration 
(1\%) of DSS and C. rodentium infection. To our knowledge, this is the first study that demonstrated the induction of colitis in mice using the combination of a chemical and a pathogen.

\section{Conflict of Interest}

The authors declare that there is no conflict of interest.

\section{Acknowledgements}

This work was supported by the 2015 Medical Cluster R\&D Support Project through Daegu Gyeongbuk Medical Innovation Foundation funded by the Ministry of Health \& Welfare, the Republic of Korea (HT15C0002). This research was supported by Korea Mouse Phenotyping Project(2014M3A9D5A01075129) of the Ministry of Science and ICT through the National Research Foundation.

\section{References}

Alipour, M., Lou, Y., Zimmerman, D., Bording-Jorgensen, M.W., Sergi, C., Liu, J.J., and Wine, E. 2013. A balanced IL-1 $\beta$ activity is required for host response to Citrobacter rodentium infection. PLoS One 8, e80656.

Andres, P.G. and Friedman, L.S. 1999. Epidemiology and the natural course of inflammatory bowel disease. Gastroenterol. Clin. North Am. 28, 255-281.

Axelsson, L.G., Landström, E., Goldschmidt, T.J., Grönberg, A., and Bylund-Fellenius, A.C. 1996. Dextran sulfate sodium (DSS) induced experimental colitis in immunodeficient mice: effects in $\mathrm{CD}^{+}{ }^{-}$-cell depleted, athymic and NK-cell depleted SCID mice. Inflamm. Res. 45, 181-191.

Bauer, C., Duewell, P., Mayer, C., Lehr, H.A., Fitzgerald, K.A., Dauer, M., Tschopp, J., Endres, S., Latz, E., and Schnurr, M. 2010. Colitis induced in mice with dextran sulfate sodium (DSS) is mediated by the NLRP3 inflammasome. Gut 59, 1192-1199.

Casati, J. and Toner, B.B. 2000. Psychosocial aspects of inflammatory bowel disease. Biomed. Pharmacother. 54, 388-393.

Chassaing, B., Aitken, J.D., Malleshappa, M., and Vijay-Kumar, M. 2014. Dextran sulfate sodium (DSS)-induced colitis in mice. Curr. Protoc. Immunol. 104, Unit 15.25.

Deng, W., Li, Y., Vallance, B.A., and Finlay, B.B. 2001. Locus of enterocyte effacement from Citrobacter rodentium: sequence analysis and evidence for horizontal transfer among attaching and effacing pathogens. Infect. Immun. 69, 6323-6335.

Eckmann, L. 2006. Animal models of inflammatory bowel disease: lessons from enteric infections. Ann. N. Y. Acad. Sci. 1072, 28-38.

Flynn, J.L., Chan, J.M., Triebold, K.J., Dalton, D.K., Stewart, T.A., and Bloom, B.R. 1993. An essential role for interferon gamma in resistance to Mycobacterium tuberculosis infection. J. Exp. Med. 178, 2249-2254.

Gibson, D.L., Ma, C., Rosenberger, C.M., Bergstrom, K.S., Valdez, Y., Huang, J.T., Khan, M.A., and Vallance, B.A. 2008. Toll-like receptor 2 plays a critical role in maintaining mucosal integrity during Citrobacter rodentium-induced colitis. Cell. Microbiol. 10, 388-403.

Hanauer, S.B. 2006. Inflammatory bowel disease: epidemiology, pathogenesis, and therapeutic opportunities. Inflamm. Bowel Dis. 12 Suppl 1, S3-S9.

Hirono, I., Kuhara, K., Yamaji, T., Hosaka, S., and Golberg, L. 1983.
Carcinogenicity of dextran sulfate sodium in relation to its molecular weight. Cancer Lett. 18, 29-34.

Hoshi, O., Iwanaga, T., and Fujino, M.A. 1996. Selective uptake of intraluminal dextran sulfate sodium and senna by macrophages in the cecal mucosa of the guinea pig. J. Gastroenterol. 31, 189198.

Iwanaga, T., Hoshi, O., Han, H., and Fujita, T. 1994. Morphological analysis of acute ulcerative colitis experimentally induced by dextran sulfate sodium in the guinea pig: some possible mechanisms of cecal ulceration. J. Gastroenterol. 29, 430-438.

Kim, J.J., Shajib, M.S., Manocha, M.M., and Khan, W.I. 2012. Investigating intestinal inflammation in DSS-induced model of IBD. J. Vis. Exp. 60, e3678.

Kitajima, S., Takuma, S., and Morimoto, M. 2000. Histological analysis of murine colitis induced by dextran sulfate sodium of different molecular weights. Exp. Anim. 49, 9-15.

Knod, J.L., Crawford, K., Dusing, M., and Frischer, J.S. 2014. Mouse strain influences angiogenic response to dextran sodium sulfate-induced colitis. J. Surg. Res. 190, 47-54.

Kwon, K.H., Murakami, A., Tanaka, T., and Ohigashi, H. 2005. Dietary rutin, but not its aglycone quercetin, ameliorates dextran sulfate sodium-induced experimental colitis in mice: attenuation of pro-inflammatory gene expression. Biochem. Pharmacol. 69, 395-406.

Luperchio, S.A. and Schauer, D.B. 2001. Molecular pathogenesis of Citrobacter rodentium and transmissible murine colonic hyperplasia. Microbes Infect. 3, 333-340.

Maaser, C., Housley, M.P., Iimura, M., Smith, J.R., Vallance, B.A., Finlay, B.B., Schreiber, J.R., Varki, N.M., Kagnoff, M.F., and Eckmann, L. 2004. Clearance of Citrobacter rodentium requires B cells but not secretory immunoglobulin A (IgA) or IgM antibodies. Infect. Immun. 72, 3315-3324.

MacDonald, T.T., Frankel, G., Dougan, G., Goncalves, N.S., and Simmons, C. 2003. Host defences to Citrobacter rodentium. Int. J. Med. Microbiol. 293, 87-93.

Moore, K.W., de Waal Malefyt, R., Coffman, R.L., and O'Garra, A. 2001. Interleukin-10 and the interleukin-10 receptor. Annu. Rev. Immunol. 19, 683-765.

Mundy, R., MacDonald, T.T., Dougan, G., Frankel, G., and Wiles, S. 2005. Citrobacter rodentium of mice and man. Cell. Microbiol. 7, 1697-1706.

Ohkusa, T., Okayasu, I., Tokoi, S., Araki, A., and Ozaki, Y. 1995. Changes in bacterial phagocytosis of macrophages in experimental ulcerative colitis. Digestion 56, 159-164.

Okayasu, I., Hatakeyama, S., Yamada, M., Ohkusa, T., Inagaki, Y., and Nakaya, R. 1990. A novel method in the induction of reliable experimental acute and chronic ulcerative colitis in mice. Gastroenterology 98, 694-702.

Ouyang, N., Zhu, C., Zhou, D., Nie, T., Go, M.F., Richards, R.J., and Rigas, B. 2012. MC-12, an annexin A1-based peptide, is effective in the treatment of experimental colitis. PLoS One 7, e41585.

Perše, M. and Cerar, A. 2012. Dextran sodium sulphate colitis mouse model: traps and tricks. J. Biomed. Biotechnol. 2012, 718617.

Randhawa, P.K., Singh, K., Singh, N., and Jaggi, A.S. 2014. A review on chemical-induced inflammatory bowel disease models in rodents. Korean J. Physiol. Pharmacol. 18, 279-288.

Rath, H.C., Herfarth, H.H., Ikeda, J.S., Grenther, W.B., Hamm, T.E. Jr., Balish, E., Taurog, J.D., Hammer, R.E., Wilson, K.H., and Sartor, R.B. 1996. Normal luminal bacteria, especially bacteroides species, mediate chronic colitis, gastritis, and arthritis in HLA-B27/human beta2 microglobulin transgenic rats. $J$. Clin. Invest. 98, 945-953.

Ryu, S.H., Park, J.H., Choi, S.Y., Jeon, H.Y., Park, J.I., Kim, J.Y., Ham, S.H., and Choi, Y.K. 2016. The probiotic Lactobacillus prevents Citrobacter rodentium-induced murine colitis in a TLR2dependent manner. J. Microbiol. Biotechnol. 26, 1333-1340.

Sang, L., Chang, B., Zhu, J., Yang, F., Li, Y., Jiang, X., Sun, X., Lu., C., 
and Wang, D. 2016. Dextran sulfate sodium-induced acute experimental colitis in C57BL/6 mice is mitigated by selenium. Int. Immunopharmacol. 39, 359-368.

Scheller, J., Chalaris, A., Schmidt-Arras, D., and Rose-John, S. 2011. The pro-and anti-inflammatory properties of the cytokine interleukin-6. Biochim. Biophys. Acta 1813, 878-888.

Simmons, C.P., Goncalves, N.S., Ghaem-Maghami, M., Bajaj-Elliott, M., Clare, S., Neves, B., Frankel, G., Dougan, G., and MacDonald, T.T. 2002. Impaired resistance and enhanced pathology during infection with a noninvasive, attaching-effacing enteric bacterial pathogen, Citrobacter rodentium, in mice lacking IL-12 or IFN- $\gamma$. J. Immunol. 168, 1804-1812.
Wu, X., Vallance, B.A., Boyer, L., Bergstrom, K.S., Walker, J., Madsen, K., O'Kusky, J.R., Buchan, A.M., and Jacobson, K. 2008. Saccharomyces boulardii ameliorates Citrobacter rodentium-induced colitis through actions on bacterial virulence factors. Am. J. Physiol. Gastrointest. Liver Physiol. 294, G295-G306.

Yamada, M., Ohkusa, T., and Okayasu, I. 1992. Occurrence of dysplasia and adenocarcinoma after experimental chronic ulcerative colitis in hamsters induced by dextran sulphate sodium. Gut 33, 1521-1527.

Yu, D., Zhu, H., Liu, Y., Cao, J., and Zhang, X. 2009. Regulation of proinflammatory cytokine expression in primary mouse astrocytes by coronavirus infection. J. Virol. 83, 12204-12214. 